Dejan Barešić

Željko Hederić

Miralem Hadžiselimović

https://doi.org/10.21278/TOF.43101

ISSN 1333-1124

eISSN 1849-1391

\title{
EXPLORING THE POSSIBILITIES OF ADJUSTING GENSETS TO NATO REQUIREMENTS
}

\begin{abstract}
Summary
Stationary and mobile gensets are used in the Armed Forces of the Republic of Croatia. In this research, priority was given to mobile gensets which have a variety of uses. In addition to being used in the Republic of Croatia, the mobile gensets are also used in international military operations in various modes of operation. Therefore, it is necessary to be very familiar with their characteristics and the possibility of adjusting them to NATO requirements. For the purpose of the adjustment, a test was performed on a P-B40.R1 genset. A model that simulates the genset behaviour with different tested fuels was developed. The investigated genset, together with some others, was introduced into the Armed Forces pursuant to the Product Quality Protocol that was in force during the 1990s. The Protocol did not cover all requirements and recommendations for the genset operation in an international military environment. Therefore, research was conducted, which has resulted in the adjustment of the equipment and in the harmonisation of the Product Quality Protocol with the ISO standard and NATO requirements. When adjusting the materiel, special emphasis is given to defining certain genset types that are able to use the modified F-34 kerosene-type fuel instead of diesel fuel. The F-34 fuel is obtained from kerosene used in aviation; this fuel can be easily obtained in international military operations where the tendency is to use the same type of fuel for airplanes, vehicles, and diesel engine equipment. When F-34 is used as the genset fuel, the quality of the generated electrical energy might be reduced. For the purpose of bringing the output characteristics of the generator within prescribed limits, simulations were performed both with and without the tuning of the regulation parameters.
\end{abstract}

Key words: $\quad$ genset, F-34 fuel, regulator adjustment, simulations

\section{Introduction}

In the Armed Forces of the Republic of Croatia, gensets are mostly used as back-up power sources in the event of interruptions in the power supply from stationary power systems. To a lesser extent, they are used to power energy consuming appliances on isolated training ranges and storage areas. A larger number of gensets are mostly stored as strategic reserves employed in the event that the stationary power system is damaged due to natural disasters or other threats. In general, gensets are rarely used and it can be deemed that they are not worn out and that their service life has been extended in comparison to the materiel in 
constant use. Some of the gensets are those of the Yugoslav People's Army, while some were procured during and after the Croatian Homeland War. This is the reason why today there are numerous different types in the Croatian Army inventory. The gensets of the Yugoslav People's Army were manufactured according to the strict military regulations in force at that time and were introduced into the Armed Forces according to Product Quality Protocols [1]. Various types of gensets, characterised by various degrees of reliability, were also procured during and after the Homeland War. The requirements regarding their characteristics were defined by various standards.

With the accession of Croatia to NATO and its participation in international military missions, additional requirements and recommendations emerged for the purpose of increasing the operability of military equipment.

Gensets were studied and sorted in order to reduce the number of various types and to increase the operability of the equipment in international military operations. That provided a detailed insight into the status and capabilities of the gensets. In other words, types of gensets that meet certain criteria were defined. Specific types of gensets that fulfil the strictest criteria and that can be used in international military operations were identified; they are followed by the types that fulfil less strict criteria for the needs of the Armed Forces in the Republic of Croatia, and then by the types that fulfil the least strict criteria for gensets to be used to aid civilian institutions in the event of natural disasters.

The study of the technical documentation of various types of gensets resulted in the finding that the genset with the P-B40.R1 type designation belongs to the group of acceptable mobile gensets that meet to the greatest extent the requirements of the Product Quality Protocol, the ISO standard, and NATO recommendations $[1,2,3]$. Subsequently, the tests and operation simulation of the P-B40.R1 genset were done.

The Republic of Croatia adopted a certain number of goals established by the NATO partners, including the "Single Fuel Concept" [4]. The goal of this concept is that all aircraft, vehicles, and diesel -engine-driven equipment intended for use in NATO-led operations should be able to function using the F-34 fuel. This fuel is based on the kerosene used as a fuel in civil aviation, designated Jet A-1 or JP-8 in the USA [5]. Research conducted thus far has revealed certain advantages and disadvantages of the F-34 fuel in comparison to diesel fuel. A decrease in power when using kerosene to power diesel engines is a known fact. This can have negative side effects during the operation of certain types of gensets, where the rotational speed is decreased under rated loads, which adversely affects the output voltage frequency [6].

Special attention is given to the problem of selecting the right type of gensets and the right mode of operation when the F-34 kerosene-type fuel is used. The aim is to avoid unwanted side effects and damages to the connected energy consuming appliances. To determine with certainty the possibility of using the F-34 kerosene-type fuel for a P-B40.R1 genset, a test and a simulation of the genset voltage dip and the frequency drop were performed in the Matlab Simulink program package. The results obtained via the simulation, such as the voltage dip and frequency drop, indicate that changes in the regulation parameters (delayed fuel injection) of an internal combustion engine can have a beneficial effect on the output characteristics of the generator, i.e. on the voltage dip and frequency drop.

\section{Performing the test on a Končar P-B40.R1 genset}

A Končar P-B40.R1 genset, whose technical characteristics are shown in Table 1, was tested in this study. The P-B40.R1 genset is composed of a three-phase, synchronous, brushless generator [7] and a four-stroke diesel engine with direct fuel injection. A specific feature of this genset is the fact that the power of the engine is significantly lower than the power of the generator. Thus, the declared power of the genset as a whole is determined by 
the engine power. The dual purpose of the test was to adjust the P-B40.R1 genset for the use of the F-34 fuel and to create a universal methodology for conducting tests of various types of gensets. Due to the complexity and the extent of the procedures, the first step was to perform a test for defining the genset behaviour while stationary. In this condition, the voltage dip and frequency drop were observed under continuous load, that is, the differences in voltage and in frequency were observed between an unloaded and a loaded genset. Thus, the transitional phenomena occurring at the moment of inclusion of the load were not observed. A genset model for predicting dynamic changes was created in the Matlab Simulink program package; the model was used to track and study dynamic changes in voltage and frequency that occur under impact loads.

Table 1 Technical characteristics of the P-B40.R1 genset

\begin{tabular}{|l|c|c|c|}
\hline & Genset & Engine & Generator \\
\hline Type designation & P-B40.R1 & T3L912 & SGB 200 SM-4 \\
\hline Permanent power & $25 \mathrm{kVA}$ & $26 \mathrm{~kW}$ & $40 \mathrm{kVA}$ \\
\hline Rotational speed & $1500 \mathrm{~min}^{-1}$ & $1500 \mathrm{~min}^{-1}$ & $1500 \mathrm{~min}^{-1}$ \\
\hline Voltage & $3 \times 400 / 231 \mathrm{~V}$ & - & $3 \times 400 / 231 \mathrm{~V}$ \\
\hline Current & $36.1 \mathrm{~A}$ & - & $57.7 \mathrm{~A}$ \\
\hline Frequency & $50 \mathrm{~Hz}$ & - & $50 \mathrm{~Hz}$ \\
\hline
\end{tabular}

Table 2 shows the values of voltage, rotational speed, and frequency obtained by performing a test using two different types of fuel, eurodiesel (ED) and kerosene-type fuel (F-34). These values are important for the classification in accordance with the Product Quality Protocol and the ISO 8528-5 standard [8]. During the test, the consumption of the kerosene-type fuel was higher than that of diesel fuel [9]. In addition, several other parameters were determined, none of them essential to this study, but they were useful in the continuation of the research conducted on gensets.

Table 2 Dependence of voltage and rotational speed on the value of the connected load

\begin{tabular}{|c|c|c|c|c|c|c|}
\hline \multirow{2}{*}{$\mathrm{P}_{\mathrm{n}}[\%]$} & \multicolumn{3}{|c|}{$\mathrm{U}[\mathrm{V}]$} & \multicolumn{2}{c|}{$\mathrm{n}[1 / \mathrm{min}]$} & \multicolumn{2}{c|}{$\mathrm{f}[\mathrm{Hz}]$} \\
\cline { 2 - 7 } & $\mathrm{ED}$ & $\mathrm{F}-34$ & $\mathrm{ED}$ & $\mathrm{F}-34$ & $\mathrm{ED}$ & $\mathrm{F}-34$ \\
\hline 0 & 400 & 400 & 1542 & 1548 & 51.4 & 51.8 \\
\hline 30 & 399 & 398 & 1525 & 1527 & 50.6 & 51.0 \\
\hline 50 & 396 & 397 & 1518 & 1521 & 50.5 & 50.6 \\
\hline 75 & 395 & 396 & 1510 & 1514 & 50.2 & 50.4 \\
\hline 100 & 394 & 395 & 1500 & 1508 & 50.0 & 50.2 \\
\hline 125 & 393 & 393 & 1499 & 1495 & 50.0 & 49.8 \\
\hline 139 & 392 & - & 1495 & - & 49.8 & - \\
\hline
\end{tabular}


The increased consumption of the kerosene-type fuel is due to its lower energy value and lower cetane number [10]. Together with the conducted test results, the power and torque characteristics of the engine have a significant role in determining the model parameters. Figure 1 shows the characteristics of a F3L912 engine, copied from the catalogue of Deutz 912 series engines [11]. In accordance with the licence for the aforementioned engine, an engine for the P-B40.R1 genset was produced, bearing the designation T3L912 and having the same engine characteristics.

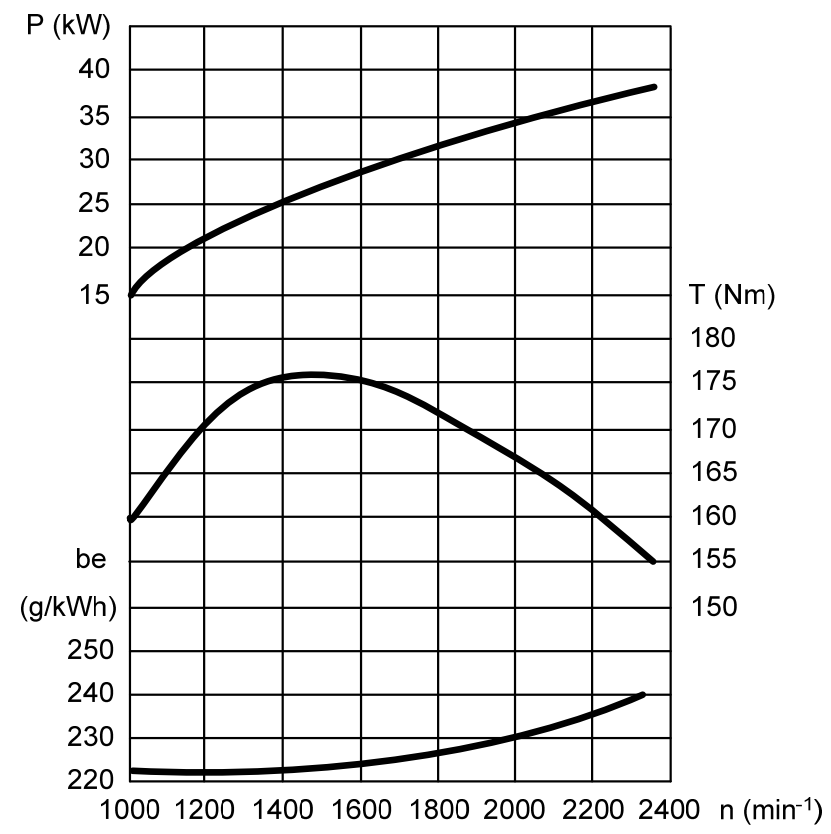

Fig. 1 Diagram showing the power, torque, and specific fuel consumption $\left(b_{\mathrm{e}}\right)$ of the 3L912 series diesel engine

\section{Simulation of the genset operation and a methodology of adjusting the regulator for using various types of fuel}

Previous studies have proved that the use of the F-34 kerosene-type fuel causes a delay in the diesel engine ignition $[12,13,14,15]$. In other words, the period between the start of fuel injection and the start of combustion is prolonged. The use of the kerosene-type fuel increases the instability of diesel engine operation [16], which affects the quality of the generated electrical energy. This effect is especially noticeable during the rated (nominal) loading and unloading of the generator.

Generators driven by turbocharged engines are even more sensitive than generators with naturally aspirated engines, which mean that they have longer reaction times in the event of sudden loads [17]. This is manifested by increased voltage dips and swells and by variation in frequency. In order to limit the dynamic changes occurring in the event of sudden loads and to bring them within allowed limits, an elaborated model (Figure 2) was developed; the simulation results would be used for tuning the genset parameters and for adjusting the rotational speed regulator of the diesel engine and the voltage regulator of the synchronous generator in real-world examples. The model does not directly include the type of fuel. Instead, using the known difference in cetane numbers of the two tested fuels and the determined lower power of the engine, the engine delay time $T_{\mathrm{d}}$ [15] is adjusted. The focus here is not on determining the exact amount of delay time $T_{\mathrm{d}}$, but on inspecting how a reduced delay time $T_{\mathrm{d}}$ affects the output characteristics of the genset (voltage and frequency) when a fuel with a lower cetane number is used. 
The diesel engine and the rotational speed regulator are described by differential equations [18]:

- Differential equation for the engine

$$
J_{d m} \frac{\mathrm{d} \omega}{\mathrm{d} t}=M_{m}\left(g_{c}, p_{k}, \omega\right)-M_{f}(\omega, N)
$$

- Differential equation for the rotational speed regulator

$$
\begin{aligned}
& T_{1}^{2}(\omega, h) \frac{\mathrm{d}^{2} g_{c}}{\mathrm{~d} t}+T_{2}(\omega, h) \frac{\mathrm{d} g_{c}}{\mathrm{~d} t}+k_{M}(\omega, h) \Delta g_{c}= \\
& =k_{1}(h) \Delta h+k_{2}(\omega, h) \Delta \omega
\end{aligned}
$$

where:

$$
k_{1}(h)=\frac{g_{c 0}}{h_{0}}, \quad k_{2}(\omega, h)=\Theta(\omega, h) \frac{g_{c 0}}{h_{0}}
$$

The research conducted in [19] showed that the assumption about the ratio of the engine torque to the amount of injected fuel can be acceptable. As a result, equations 1 and 2 can be composed in the following form, with the introduction of initial conditions:

$$
\begin{aligned}
& \frac{\mathrm{d} \omega}{\mathrm{d} t}=\frac{1}{J_{d g}}\left(M_{m}-M_{e l}\right) \\
& \frac{\mathrm{d} m_{T}}{\mathrm{~d} t}=\Delta m_{T} \\
& \frac{\mathrm{d} \Delta m_{T}}{\mathrm{~d} t}=\left(-T_{2} \Delta m_{T}-m_{T}-k \omega+k \omega_{0}+m_{T 0}\right) \frac{1}{T_{1}^{2}}
\end{aligned}
$$

The differential equations for the synchronous generator in d-q coordinates are as follows:

$$
\begin{aligned}
& -u_{d}=\frac{\mathrm{d} \psi_{d}}{\mathrm{~d} t}-\psi_{q} \frac{\mathrm{d} y}{\mathrm{~d} t}+r i_{d} \\
& -u_{q}=\psi_{d} \frac{\mathrm{d} y}{\mathrm{~d} t}+\frac{\mathrm{d} \psi_{q}}{\mathrm{~d} t}+r i_{q} \\
& 0=\frac{\mathrm{d} \psi_{D}}{\mathrm{~d} t}+r_{D} i_{D} \\
& u_{1}=\frac{\mathrm{d} \psi_{1}}{\mathrm{~d} t}+r_{1} i_{1} \\
& J_{D G} \frac{\mathrm{d} y^{2}}{\mathrm{~d} t}=M_{m}-M_{e l m}
\end{aligned}
$$

where: 


$$
\begin{array}{ll}
\psi_{d}=x_{d} i_{d}+e_{q}+i_{D} x_{a d} ; & \psi_{q}=x_{q} i_{q}+i_{Q} x_{a q} \\
\psi_{D}=i_{d} x_{a d}+e_{q}+i_{D} x_{D} ; & \psi_{Q}=i_{q} x_{a q}+i_{Q} x_{Q} \\
\psi_{1}=i_{d} x_{a d}+i_{1} x_{1}+i_{D} x_{a d} ; & M_{e l m}=-\psi_{d} i_{q}+\psi_{q} i_{d}
\end{array}
$$

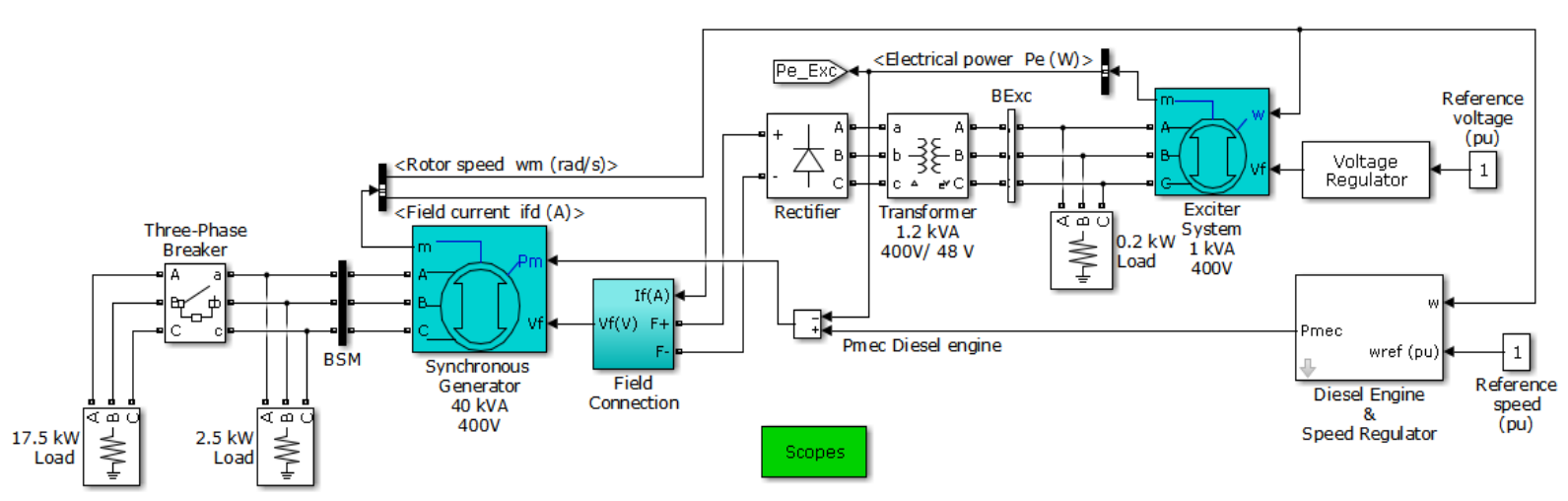

Fig. 2 A model created in MATLAB Simulink that was used in the simulation

The parameters stated in the technical documentation and the results of performed tests were used to set up the model shown in Figure 2. Additional parameters, which had not been obtained from the technical documentation or the performed tests, were obtained by mathematical interpolation of the existing values of larger and smaller genset units that can be found in the Contents of the Matlab Simulink program package. First, the parameters indicated in the technical documentation $[11,20]$ (power, rotational speed, current, frequency, and voltage of the excitation circuit) were used for calibrating the model.

Then, additional parameters required for the presented model were obtained through the test performed in [9]; for example, the test results showed that the power of the diesel engine was reduced by approx. $10 \%$ when the kerosene-type fuel was used. Tests with diesel fuel were also performed on a similar genset $[18,19]$ resulting in more additional parameters, such as moments of inertia and other parameters of a synchronous generator (reactances, winding resistances, time constants, etc.). The parameters that could not be obtained from the indicated sources were taken over from models that had already been developed in the MATLAB Simulink program package. The models were developed for larger or smaller gensets, so values of the parameters needed to be adjusted for the P-B40.R1 genset.

The goal of this simulation was to achieve solutions for obtaining the same characteristics of gensets irrespective of the type of engine fuel (diesel or F-34).

The idea is to achieve for a genset using the F-34 fuel to retain the same performances as with diesel fuel. According to their performances, gensets are classified into four groups (G1, G2, G3 and G4) [8]. Group G4 includes gensets that meet the strictest quality requirements for the generated energy, which means those with the most stable generator voltage and frequency. Group G1 allows the greatest voltage dips and frequency drops for its class.

When conducting the simulations, we put special emphasis on studying dynamic changes in voltage and frequency during sudden loading and unloading of the generator. Figure 3 shows the changes in the power of the engine $\left(P_{\mathrm{mec}}\right)$, in the rotational speed (Speed) and in the voltage of the generator (Voltage) in a period of 10 seconds. The impact loading occurs after 3 seconds, while the unloading occurs after 6 seconds. The values of the simulation shown in Figure 3 refer to diesel fuel and match the values shown in graphs 
depicted in ISO 8528. The results of the simulations occurring in the first second are not essential and can be neglected.
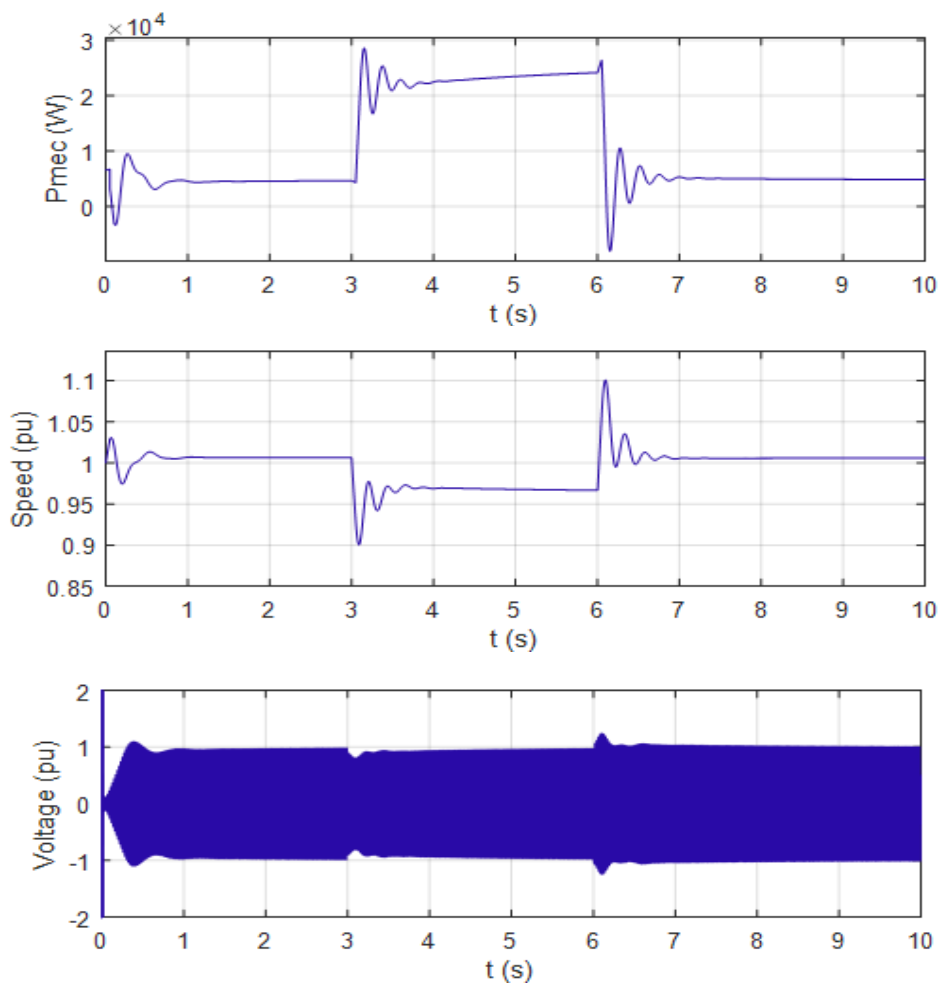

Fig. 3 Dynamic changes in the engine power, the engine speed, and the generator voltage when using diesel fuel

In the figures that follow (4 to 13), the results of simulations performed for the two types of fuel are compared.

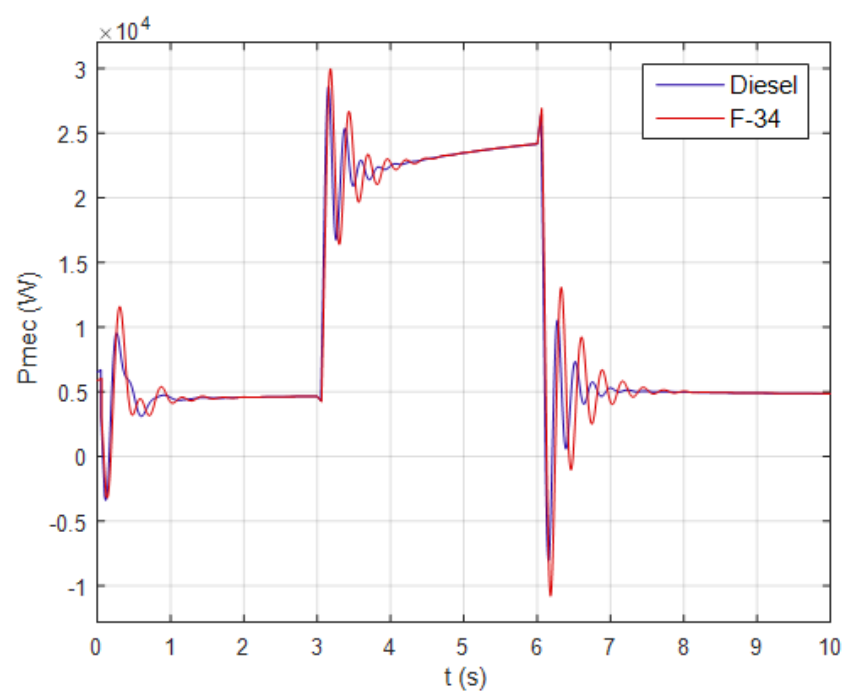

Fig. 4 Engine power for two different types of fuel, without injection tuning

Figure 4 shows that greater oscillations in the developed engine power occur when the F-34 fuel is used. Figure 5 shows a drop in the rotational speed occurring after the first 3 seconds from the start of the simulation, at the moment of loading of the generator, and a rise in the rotational speed occurring after 6 seconds, at the moment of unloading of the generator. It is evident that sharper drops and steeper rises occur when using the F-34 kerosene-type 
fuel. The analysis of the obtained results according to [8] leads to the conclusion that the drops and rises in the rotational speed exceed the allowed limits prescribed for the genset class G2 when the F-34 fuel is used. For example, for the class G2, the allowed frequency increase when a generator that was loaded at $100 \%$ of the rated (nominal) power is suddenly unloaded is $12 \%$. This means that the allowed change in the rotational speed in that case is also $12 \%$ maximum. The maximum allowed voltage swell upon sudden unloading is $25 \%$. This means that the genset using the F-34 fuel would in this case be classified as belonging to the lower class (G1) according to the ISO classification [8] which sets less stringent requirements for the quality of the generated electrical energy.

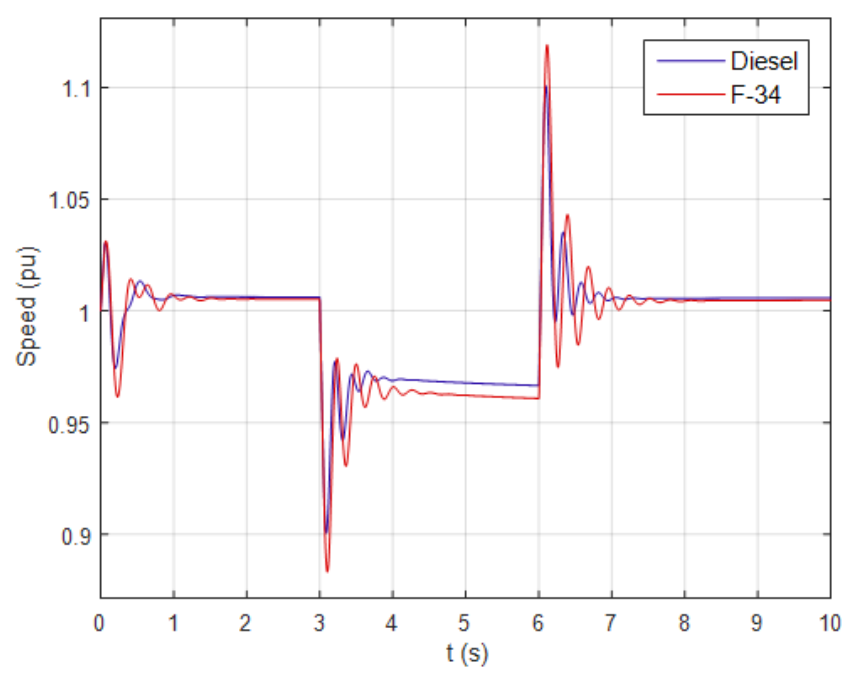

Fig. 5 Rotational speed of the generator for two different types of fuel, without injection tuning

The same happens with voltage dips and swells, as shown in Figure 6 and 7. Figure 6 shows that the voltage dip exceeds $20 \%$ when using the F-34 fuel, meaning that, at a specific moment, the voltage drops below $0.8 \mathrm{~V}$, which is the borderline for the $\mathrm{G} 2$ class. The same goes for voltage swells, as shown in Figure 7, where they exceed $25 \%$ of the set value when using the F-34 fuel.

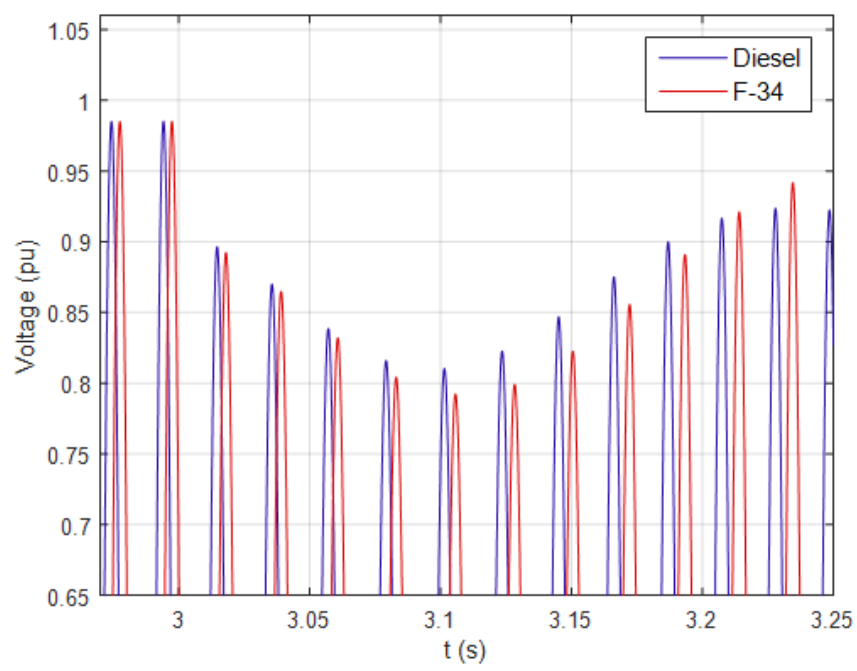

Fig. 6 Generator voltage sag during impact loading for two different types of fuel, without injection tuning 


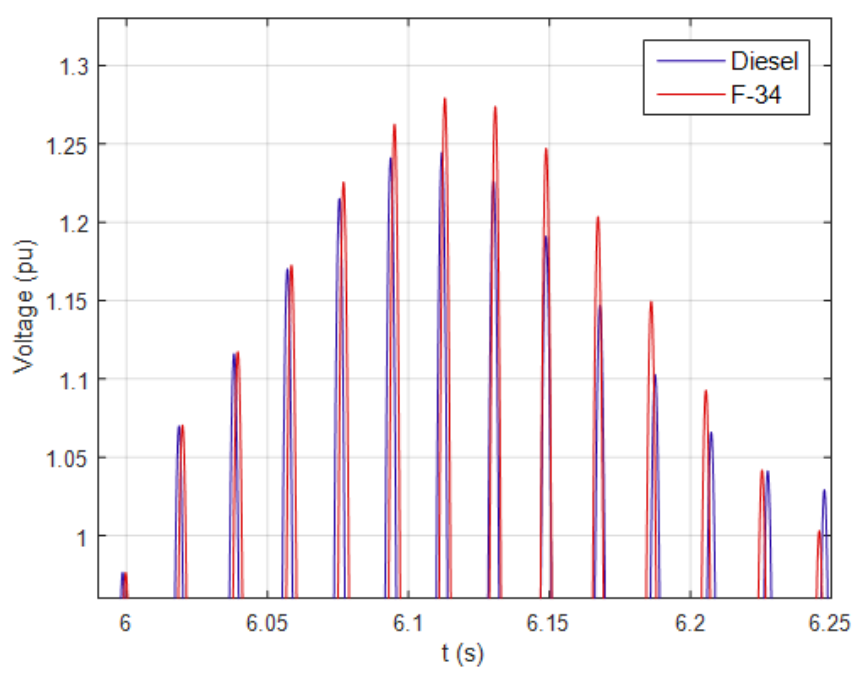

Fig. 7 Generator voltage swells during sudden unloading for two different types of fuel, without injection tuning

In order to achieve the best quality of the electrical energy generated when using the kerosene-type fuel, we investigated the rotational speed regulator of the engine and the voltage regulator of the generator. The research showed that by tuning the regulation parameters, it was possible to obtain very similar characteristics in certain phases in testing regardless of the type of engine fuel.

In our case, the dynamic changes during impact loading and unloading of the generator represent an important phase in testing. Under rated continuous loads, it is known that greater drops occur when using the F-34 fuel, but these are within the allowed limits prescribed for the G2 class. This is best seen in Figure 5, between the fifth and the sixth second.

Seeing as there are different options for tuning the regulator, the goal is to choose the simplest method that can be applied to various types of gensets.

By studying the F-34 kerosene-type fuel, a lower cetane number was determined. This can lead to a more difficult engine start at lower temperatures and to an ignition delay in the engine cylinder. Taking into account the results of the research conducted thus far, a simulation using different values for the engine delay $T_{\mathrm{d}}$ was also conducted, alongside other simulations. In that simulation, it was determined that injecting the fuel earlier can significantly affect the characteristics of the generator frequency and voltage during impact loading and unloading. By tuning the injection, that is, by shortening the delay time $T_{\mathrm{d}}$ by $10 \%$ when conducting the simulation for the kerosene-type fuel, the values shown in Figure 8 to 11 were obtained. These values show that the rises, drops and oscillations were greatly reduced, that is, that they were equal for both types of fuel. In that case, the genset is sorted into the same class regardless of the type of the fuel used.

This procedure is easy to adopt and can be used for various injection systems. This can be done by rotating the high-pressure pump in an older system, or by changing the electric parameters of the rotational speed regulator of the engine in newer systems. 


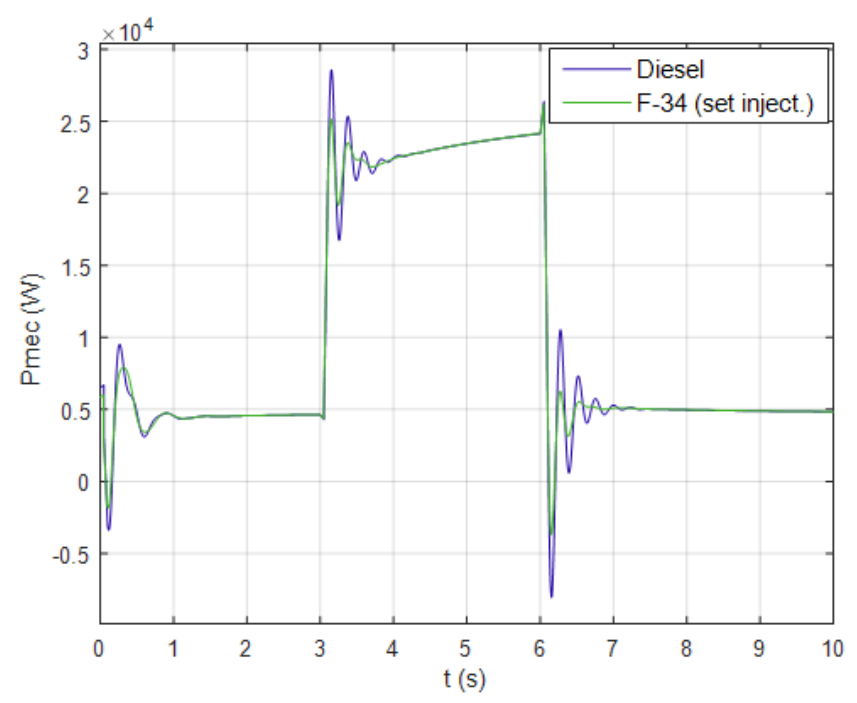

Fig. 8 Engine power developed with two different types of fuel used and with the injection tuned

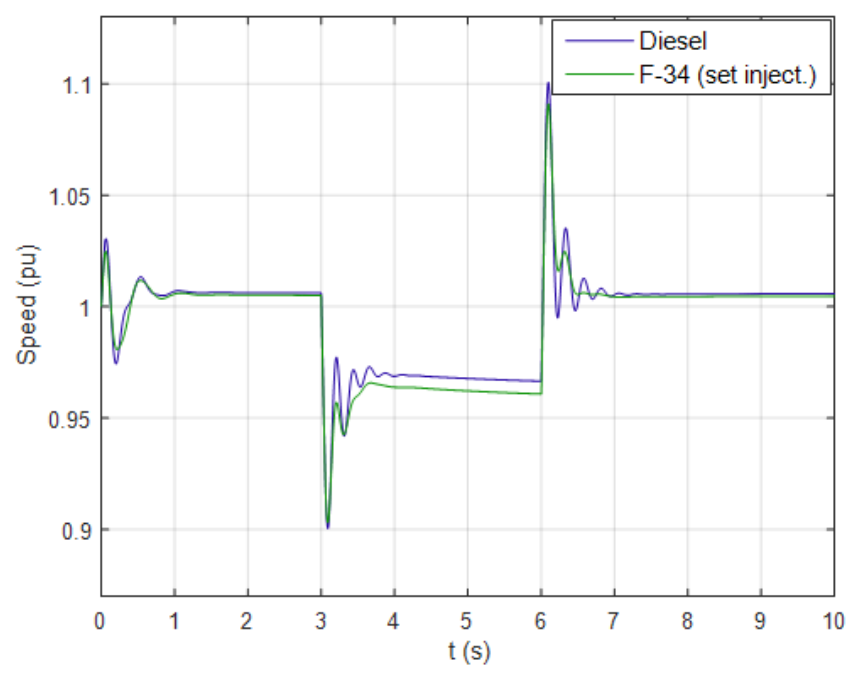

Fig. 9 Generator rotational speed with two different types of fuel used and with the injection tuned

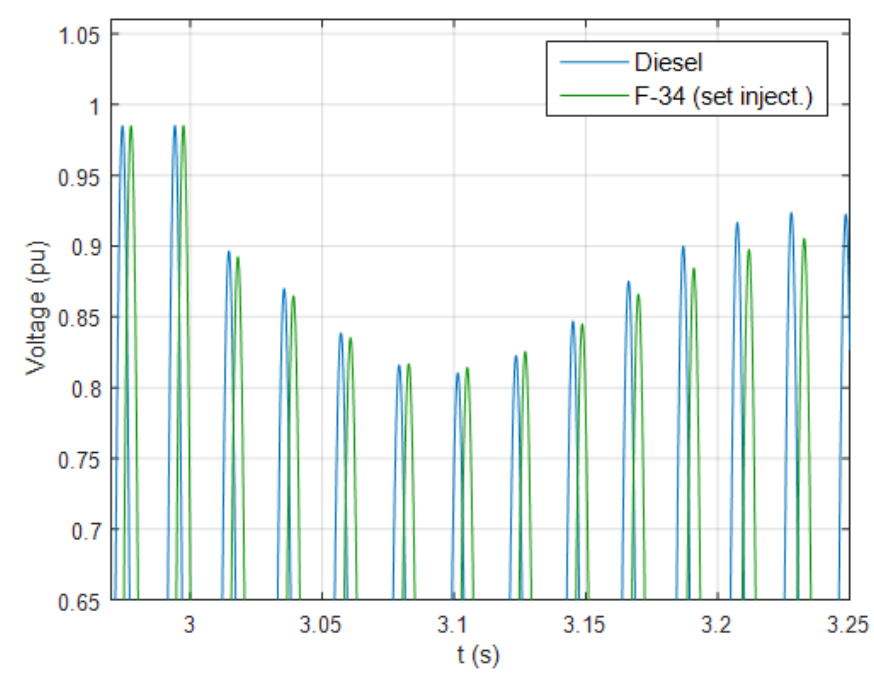

Fig. 10 Generator voltage dip during impact loading for two different types of fuel, with the injection tuned 


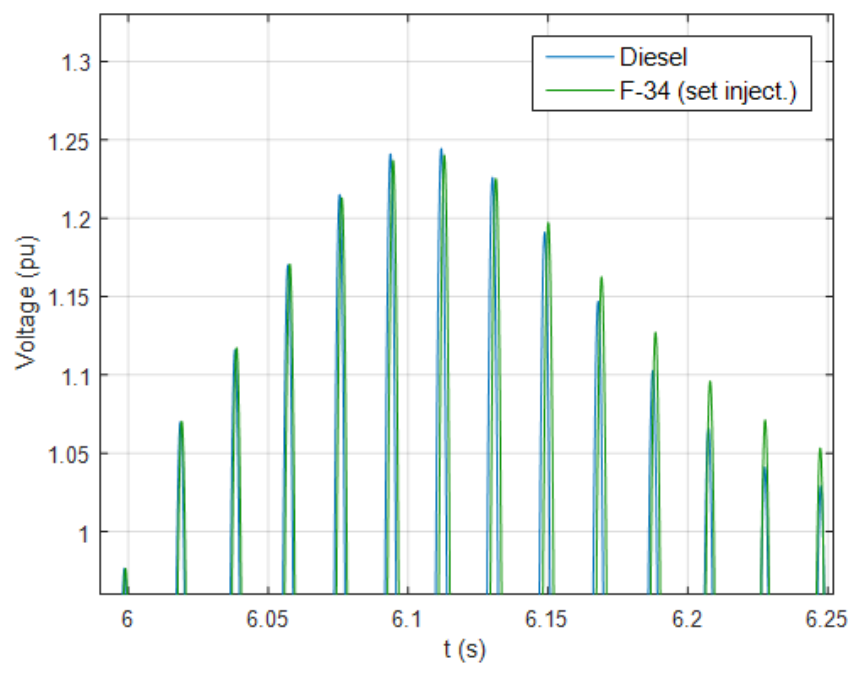

Fig. 11 Generator voltage swell during sudden unloading for two different types of fuel, with the injection tuned

The research conducted thus far has shown that the required rotation of the highpressure pump to an earlier phase does not have a significant negative impact on the operation of the engine. The same engines are also often used in various driven machines where they rotate at increased speeds and are tuned for an earlier ignition stage. This also confirms the possibility of operation with a somewhat earlier ignition stage without causing damage to the engine.

Figure 12 and 13 show an enlarged view of the rotational speed decrease and increase occurring during the impact loading and unloading of the generator, respectively. The blue curve, representing the generator using diesel fuel and the factory settings of the engine, was obtained via the simulation first. Afterwards, the red curve, representing the generator using the F-34 fuel and the factory settings of the engine, was obtained. Here, it is evident that the rotational speed sharply decreases (Figure 12) and increases (Figure 13). One can also note delayed decreases and increases occurring due to the later ignition of the F-34 fuel. In order to avoid the bigger decreases and increases that occur when the F-34 fuel is used, the engine delay time was tuned. The green curve was thus obtained. The curve confirms that it is possible to reduce the rotational speed decreases and increases that occur when using the F-34 fuel and to bring them within the limits defined by ISO 8528 by tuning the fuel injection time.

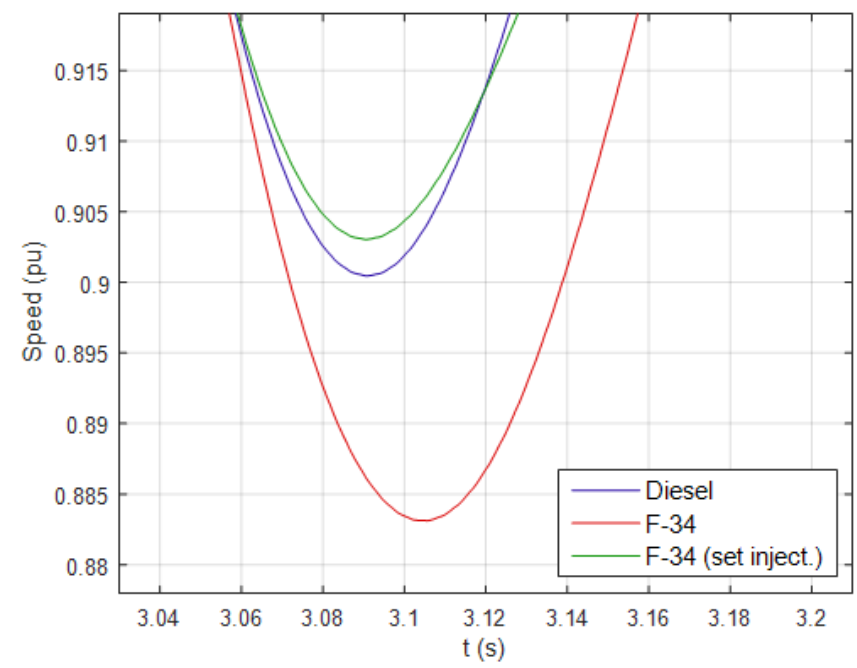

Fig. 12 Rotational speed decrease before and after the tuning of the fuel injection system 


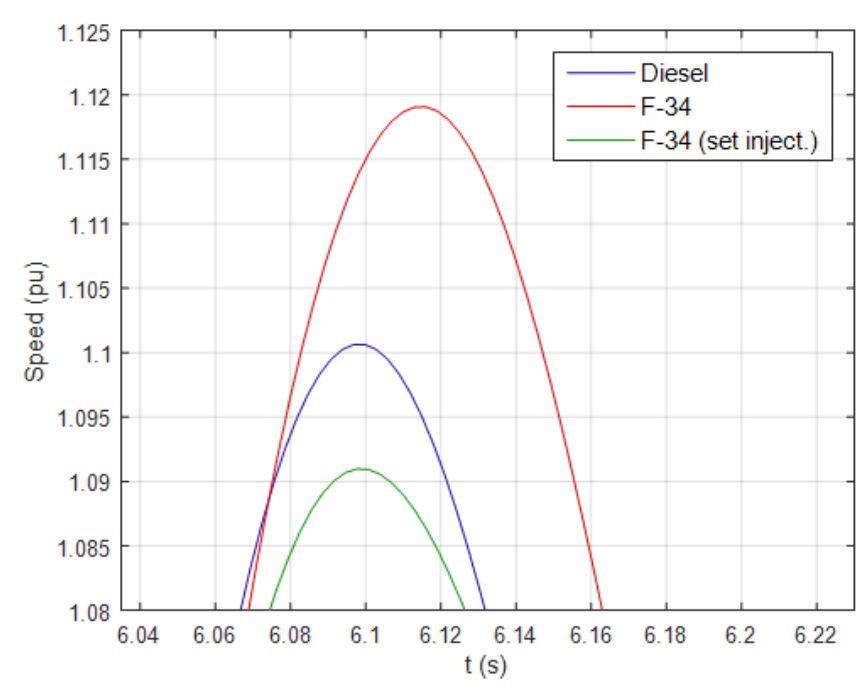

Fig. 13 Rotational speed increase before and after the tuning of the fuel injection system

\section{Conclusions}

The research conducted thus far indicates that oscillations in engine power and rotational speed occur during impact loading and unloading of the generator. This directly affects the increase in the voltage dips and swells and the variation in frequency.

The decreases and increases are more pronounced when the F-34 kerosene-type fuel is used. The conducted simulations lead to the conclusion that the oscillations, which occur due to the lower energy value and the inferior cetane number of the F-34 fuel, are decreased by changing the internal combustion engine regulation parameters. The simplest method that is applicable to most gensets is to tune the injection system, that is, to decrease the engine delay time. This can compensate for the more dramatic dynamic changes occurring when the F-34 fuel is used than in the case when diesel fuel is used.

The emphasis of this research was placed on the dynamic changes in voltage and frequency since, in this case, they are most critical and they define the genset class. By bringing the dynamic changes occurring when the F-34 fuel is used within certain limits, the application of the gensets is significantly simplified in various modes of operation,; this means that the gensets are brought to a state in which they meet the requirements of the Product Quality Protocol and the ISO 8528 standard for both types of fuel.

In the continuation of this research, experimental tests will be performed on various types of gensets, and the results will be compared with the results of the simulations.

\section{Nomenclature}

$$
\begin{array}{ll}
g_{\mathrm{c}} & - \text { amount of fuel } \\
g_{\mathrm{c} 0} & - \text { amount of fuel at idle } \\
\mathrm{h}_{0} & - \text { position of fuel lever at idle } \\
i_{\mathrm{D}}, i_{\mathrm{Q}}, r_{\mathrm{D}}, r_{\mathrm{Q}} & - \text { currents and resistances in the equivalent damper winding } \\
J_{\mathrm{dg}} & - \text { moment of inertia of a diesel set } \\
J_{\mathrm{dm}} & - \text { moment of inertia of a diesel engine } \\
k & - \text { static gain } \\
k_{1} & - \text { engine gain factor }
\end{array}
$$




$\begin{array}{ll}k_{2} & - \text { actuator gain factor } \\ M_{\mathrm{elm}} & - \text { electromagnetic moment of a synchronous generator } \\ M_{\mathrm{m}}, M_{\mathrm{f}} & - \text { engine torque and load moment } \\ m_{\mathrm{T}} & - \text { propulsion engine torque } \\ m_{\mathrm{T} 0} & - \text { moment of propulsion engine in initial condition } \\ N & - \text { power supply system load } \\ p_{\mathrm{k}} & - \text { air pressure in the intake manifold } \\ r, r_{1} & - \text { active resistance of the armature and excitation winding } \\ t & - \text { time } \\ T_{1} & - \text { regulator inertia time constant } \\ T_{2} & - \text { regulator damping time constant } \\ u_{\mathrm{d}}, u_{\mathrm{q}}, i_{\mathrm{d}}, i_{\mathrm{q}} & - \text { voltage and current in the longitudinal and the transverse machine axis } \\ u_{1}, i_{1} & - \text { excitation voltage and current } \\ x_{\mathrm{D}}, x_{\mathrm{Q}} & - \text { reactances of the damper winding in the } d \text { and } q \text { axes } \\ x_{\mathrm{d}}, x_{\mathrm{q}} & - \text { synchronous reactances of the armature in the } d \text { and } q \text { axes } \\ x_{\mathrm{ad}}, x_{\mathrm{aq}} & - \text { armature voltage reactances in the } d \text { and } q \text { axes } \\ x_{1}, e_{\mathrm{q}} & - \text { reactance and voltage of the excitation winding } \\ \Delta h & - \text { fuel lever shift } \\ \Delta \omega & - \text { change in rotational speed } \\ \Theta & - \text { speed regime effectiveness factor } \\ \Psi_{\mathrm{d}}, \Psi_{\mathrm{q}}, \Psi_{\mathrm{D}}, \Psi_{\mathrm{Q}} & - \text { chained flows in the longitudinal and transverse axes of the armature } \\ \Psi_{1} & \text { and equivalent damper winding } \\ & - \text { chained flow with excitation winding }\end{array}$

\section{REFERENCES}

[1] Product quality protocol „AC and DC gensets, up to $1 \mathrm{MW}$ in power“, Federal People's Defence Committee, Office for Standardization and Metrology, Belgrade, 1986.

[2] Book: NATO STANAG 4362: "Fuels for future ground equipment using compression ignition or turbine engines"

[3] NATO Logistics Handbook, Senior NATO Logisticians' Conference Secretariat NATO Headquarters Brussels, 1997.

[4] R. Spudić, K. Somek, V. Kovačević, "Single Fuel Concept for Croatian Army Ground Vehicles," Promet - Traffic \& Transportation, Vol. 20, No. 3, pp. 181-187, 2008

[5] G. Fernandes, J. Fuschetto, Z. Filipi, "Impact of Military JP-8 Fuel on Heavy Duty Diesel Engine Performance and Emissions," Proceedings of the Institution of Mechanical Engineers, Part D: Journal of Automobile Engineering, Volume 221, Issues 8, 2007. https://doi.org/10.1243/09544070jauto211

[6] K. Wadumesthrige, N. Johnson, M. Winston-Galant, "Performance and Durability of a Generator Set CI Engine using Synthetic and Petroleum Based Fuels for Military Applications," Applied Energy, Volume 87, Issues 5, 2010. https://doi.org/10.1016/j.apenergy.2009.09.015

[7] P. C. Krause, O. Wasynczuk, S. D. Sudhoff, Analysis of Electric Machinery and Drive Systems, Wiley, USA, 2013. https://doi.org/10.1002/9781118524336

[8] International Organization for Standardization, ISO 8528-1 through 8528-6, Genève, 1993

[9] D. Barešić, Ž. Hederić, M. Barukčić, "Testing of gensets with diesel engine to use a modified kerosene fuel, "EIS 2013 Proceedings, $26^{\text {th }}$ International Symposium "Electrical Engineering Symposium" Josip Lončar Days / Neven Srb (ed.).

Zagreb: Elektrotehničko društvo Zagreb, 2013. pp. 28-31 
[10] R. Cataluña, R. da Silva, "Effect of Cetane Number on Specific Fuel Consumption and Particulate Matter and Unburned Hydrocarbon Emissions from Diesel Engine," Journal of Combustion Volume 2012, 2012. https://doi.org/10.1155/2012/738940

[11] https://www.deutzpartsdirect.com/documents/deutz-specs/deutz-912-agricultural-specs.pdf (available on 1 August 2018)

[12] D. A. Rothamer, L. Murphy, "Systematic study of ignition delay for jet fuels and diesel fuel in a heavyduty diesel engine," Proceedings of the Combustion Institute, Volume 34, Issue 2, 2013. https://doi.org/10.1016/j.proci.2012.06.085

[13] P. Schihl, L. Hoogterp-Decker, "On the Ignition Behaviour of JP-8 in Military Relevant Diesel Engines," SAE International Journal of Engines Volume 4, Issues 1, 2011. https://doi.org/10.4271/2011-01-0119

[14] G. Labeckas, S. Slavinskas, "Combustion phenomenon, performance and emissions of a diesel engine with aviation turbine JP-8 fuel and rapeseed biodiesel blends," Energy Conversion and Management Volume 105, 15 November 2015. https://doi.org/10.1016/j.enconman.2015.07.065

[15] J. B. Heywood, Internal Combustion Engine Fundamentals, McGraw-Hill, New York, 1988

[16] K. Wadumesthrige, N. Johnson, M. Winston-Galant, "Performance, Durability and Stability of a Power Generator Fueled with ULSD, S-8, JP-8, and Biodiesel," SAE International Journal of Engines, 2010. https://doi.org/10.4271/2010-01-0636

[17] T. Katrašnik, V. Medica, F. Trenc, "Analysis of the dynamic response improvement of a turbocharged diesel engine driven alternating current generating set," Energy Conversion and Management Volume 46, Issues 18-19, November 2005. https://doi.org/10.1016/j.enconman.2005.03.001

[18] V. Matijević, Z. Maljković, "Impact of speed regulation of diesel engines on the dynamic characteristics of synchronous generator," Automatika, 31, 3-4, Zagreb, 1990

[19] M. Mirošević, "Dinamika brodskih elektroagregata u autonomnom pogonu," Master Thesis, Faculty of Electrical Engineering and Computing, Zagreb, Croatia, 1999

[20] P-B40 genset operation and maintenance manual, Končar-ERS, Rijeka, Croatia, 1995

Submitted: $\quad 26.02 .2018$

Accepted: $\quad 08.01 .2019$
Dejan Barešić

Croatian Military Academy "Dr. Franjo

Tuđman” Ilica 256b, 10000 Zagreb,

Croatia, e-mail: dejan.baresic@morh.hr

Željko Hederić

J.J. Strossmayer University of Osijek, Faculty of Electrical Engineering,

Computer Science and Information

Technology, Ul. kneza Trpimira 2B,

31000 Osijek, Croatia

Miralem Hadžiselimović

University of Maribor, Faculty of Energy

Technology, Hočevarjev trg 1,8270

Krško, Slovenia 